\title{
A Smartphone App for Patients With End-Stage Liver Disease Can Detect Behavioral Changes That Predict Liver-Related Events
}

\author{
Jordan Sack $^{1,2^{*}}$, MD; Todd Reid ${ }^{3,4^{*}}$, MBA, MPH, ScD; Eric Schlossberg ${ }^{4}$; Nikroo Hashemi ${ }^{1,2}$, MD, MPH \\ ${ }^{1}$ Division of Gastroenterology, Hepatology and Endoscopy, Brigham and Women's Hospital, Boston, MA, United States \\ ${ }^{2}$ Harvard Medical School, Boston, MA, United States \\ ${ }^{3}$ Harvard TH Chan School of Public Health, Boston, MA, United States \\ ${ }^{4}$ MIT Media Lab and MIT Connection Science, Cambridge, MA, United States \\ *these authors contributed equally
}

\section{Corresponding Author:}

Jordan Sack, MD

Brigham and Women's Hospital

Division of Gastroenterology, Hepatology and Endoscopy

75 Francis Street

Boston, MA

United States

Phone: 6175257743

Email: jsack@bwh.harvard.edu

\section{Abstract}

Background: Patients with end-stage liver disease have significant morbidity and mortality. The 90-day readmission rate for these patients is up to $53 \%$ at a cost of $\$ 4.45$ billion annually. Healthcare delivery for these patients is often fragmented and inadequate. Smartphone-based remote health monitoring may reduce hospitalizations by earlier detection of premonitory warning signs associated with liver-related complications. Hepatic encephalopathy which is a common cause of hospitalization and sleep disturbance and subtle/sub-clinical behavioral changes are early warning signs.

Objective: In this pilot study of patients with end-stage liver disease, we assessed the feasibility of our smartphone app to detect physiologic and behavioral changes during the 7 days prior to liver-related hospitalizations or urgent visits.

Methods: This is a prospective multicenter pilot study of patients with end-stage liver disease who were enrolled at three academic centers to receive our smartphone app for a 180-day period. English speaking patients age $\geq 18$ years who receive liver care at one of the study sites, do not actively use alcohol or drugs, have had a liver-related complication in the previous 3 months (ascites, hepatic encephalopathy, variceal bleeding, bacterial peritonitis), and own an Android smartphone with internet connectivity were eligible. The smartphone app solicits emotions daily and collects passive data on activity, sleep, and social interactions. Patients received monthly in-app questions about how many liver-related events they had over the preceding month. Surveys on sociodemographic characteristics and health status were collected at baseline, 90 days, and 180 days. Clinical data on liver-related hospitalizations or urgent visits ("events") were collected prospectively through chart review. Smartphone data on activity, sleep, social interactions, and emotions were analyzed during the 7-day period preceding a liver event and compared to the average over the study period. Statistical analyses were performed with Mann-Whitney U test.

Results: An interim analysis of the 40 enrolled patients who met all eligibility criteria found that 15 patients had 27 liver-related events during the study period. These patients were predominantly men with a median age of 56 years. $61 \%$ of these patients responded to the monthly in-app question about hospitalizations and did so with $100 \%$ accuracy. In the 7-day period prior to the event, these patients had more sleep disturbances and changes in activity score $(P=.04 ; P=.04)$. There was no statistically significant difference in social scores during the 7-day period prior to the event. Emoji selection among this group was too small for analysis.

Conclusions: The interim analysis of this pilot study suggests that passive data collected from our smartphone app can detect behavioral changes that could be used to predict liver-related events. Specifically, significant changes in smartphone activity and sleep disturbances were identified during the 7-day period prior to a liver-related event. Smartphone-based remote health monitoring appears to be feasible in this patient population and has the potential to reduce hospitalizations through early detection of early warning signs.

(iproc 2019;5(1):e15229) doi: 10.2196/15229 


\section{KEYWORDS}

app; smartphone; cirrhosis

Edited by J Brown; this is a non-peer-reviewed article. Submitted 27.06.19; accepted 13.08.19; published 02.10.19.

Please cite as:

Sack J, Reid T, Schlossberg E, Hashemi N

A Smartphone App for Patients With End-Stage Liver Disease Can Detect Behavioral Changes That Predict Liver-Related Events iproc 2019;5(1):e15229

URL: http://www.iproc.org/2019/1/e15229/

doi: $\underline{10.2196 / 15229}$

PMID:

CJordan Sack, Todd Reid, Eric Schlossberg, Nikroo Hashemi. Originally published in Iproceedings (http://www.iproc.org), 02.10.2019 This is an open-access article distributed under the terms of the Creative Commons Attribution License (https://creativecommons.org/licenses/by/4.0/), which permits unrestricted use, distribution, and reproduction in any medium, provided the original work, first published in Iproceedings, is properly cited. The complete bibliographic information, a link to the original publication on http://www.iproc.org/, as well as this copyright and license information must be included. 